\title{
Erratum: Dark Matter Interpretation of the Neutron Decay Anomaly [Phys. Rev. Lett. 120, 191801 (2018)]
}

\author{
Bartosz Fornal@ and Benjamín Grinstein $\odot$
}

(Q) (Received 7 May 2020; published 28 May 2020)

DOI: 10.1103/PhysRevLett.124.219901

We correct an error in Eq. (5) of our Letter. The coefficient of the neutron magnetic moment interaction should be $g_{n} e /\left(8 m_{n}\right)$ instead of $g_{n} e /\left(2 m_{n}\right)$. Apart from Eq. (5), only Eqs. (6), (7) and (15) are affected by this change. All other equations and the entire discussion in the Letter remain accurate as they are.

Equation (5) should read

$$
\mathcal{L}_{1}^{\mathrm{eff}}=\bar{n}\left(i \not \supset-m_{n}+\frac{g_{n} e}{8 m_{n}} \sigma^{\mu \nu} F_{\mu \nu}\right) n+\bar{\chi}\left(i \not \partial-m_{\chi}\right) \chi+\varepsilon(\bar{n} \chi+\bar{\chi} n),
$$

which implies that Eq. (6) should be

$$
\mathcal{L}_{n \rightarrow \chi \gamma}^{\mathrm{eff}}=\frac{g_{n} e}{8 m_{n}} \frac{\varepsilon}{\left(m_{n}-m_{\chi}\right)} \bar{\chi} \sigma^{\mu \nu} F_{\mu \nu} n
$$

and Eq. (7) should read

$$
\Delta \Gamma_{n \rightarrow \chi \gamma}=\frac{g_{n}^{2} e^{2}}{128 \pi}\left(1-\frac{m_{\chi}^{2}}{m_{n}^{2}}\right)^{3} \frac{m_{n} \varepsilon^{2}}{\left(m_{n}-m_{\chi}\right)^{2}} \approx \Delta \Gamma_{n}^{\exp }\left(\frac{1+x}{2}\right)^{3}\left(\frac{1-x}{1.8 \times 10^{-3}}\right)\left(\frac{\varepsilon[\mathrm{GeV}]}{3.7 \times 10^{-13}}\right)^{2} .
$$

This requires a modification of Eq. (15) to

$$
\frac{\left|\lambda_{q} \lambda_{\chi}\right|}{M_{\Phi}^{2}} \approx 2.7 \times 10^{-5} \mathrm{TeV}^{-2}
$$

Published by the American Physical Society under the terms of the Creative Commons Attribution 4.0 International license. Further distribution of this work must maintain attribution to the author(s) and the published articles title, journal citation, and DOI. 